\title{
Doppler sonography in acute renal obstruction
}

\author{
Sonali S Saboo, Sachin S Soni ${ }^{+}$, Suresh H Saboo ${ }^{++}$, Naga Ramesh Chinapuvvula, Sashidhar Kaza \\ Department of Radiodiagnosis, Yashoda Superspeciality Hospitals, Hyderabad (AP), +Department of Nephrology, MediCiti \\ Hospitals, Hyderabad (AP), ${ }^{++}$Sai Diagnostics, Jalna, Maharashtra, India
}

Correspondence: Dr. Sonali S Saboo, Flat F3, 5-9-22/60A, Adarsha Vihar Apartment, Adarsha Nagar, Hyderabad, India. E-mail: drsonalisoni@rediffmail.com

\begin{abstract}
Aims and Objectives: This prospective analysis was carried out to study the utility of Doppler waveform alterations in the diagnosis of unilateral acute renal obstruction and to study the factors affecting Doppler indices. Methods: In this prospective analysis between May 2005 and March 2006, patients presenting to the emergency medical division with symptoms of unilateral acute renal colic underwent Doppler analysis. Obstruction was confirmed by intravenous urography (IVU) or computed tomography (CT) scan. The duration of renal colic and the site and degree of obstruction were recorded. Patients with medical or surgical renal disease were excluded. Results: 40 patients (80 kidneys) satisfying the inclusion criteria were part of this analysis. The mean resistivity Index (RI) in obstructed kidneys was significantly higher than in the contralateral unobstructed kidneys $(0.72 \mathrm{Vs} 0.64 ; \mathrm{p}$ $<0.001)$. Thirty $(75 \%)$ cases had proximal while $10(25 \%)$ cases had distal obstruction with higher RI in proximal obstruction $(0.73$ Vs 0.72 ). Twenty-five (62.5\%) patients had complete, while $15(37.5 \%)$ patients had partial obstruction. The RI value in completely obstructed kidneys was significantly higher than in partially obstructed kidneys $(0.74 \mathrm{Vs} 0.70 ; p<0.05)$. Using the discriminatory value of 0.70 for obstruction, the overall sensitivity was $87.5 \%$ and specificity $90 \%$. Sensitivity was $95 \%$ and specificity $100 \%$ at a delta RI of 0.06 . The degree of obstruction altered the resistivity indices, while the site of obstruction and duration of symptoms did not. Conclusions: Doppler sonography is a useful diagnostic tool in unilateral acute renal obstruction.
\end{abstract}

Key words: Doppler sonography; resistivity indices; unilateral acute renal obstruction.

\section{Introduction}

USG remains a commonly used modality in the initial evaluation and diagnosis of renal obstruction. It can dependably detect dilatation of the urinary system proximal to the level of obstruction, which is an indirect evidence for the diagnosis. However, urinary system dilatation seen on USG has been shown to be sensitive (90\%) but not specific (65\%-84\%) in the diagnosis of renal obstruction. ${ }^{[1]}$ It has been reported ${ }^{[2]}$ that the diagnosis of obstructive uropathy may be missed by USG because pyelocaliectasis may occur late in obstructive conditions and often the findings are normal despite severe renal dysfunction.

Animal studies have shown that there is a definite rise in vascular resistance in the renal arteries when the kidney is obstructed. ${ }^{[3]}$ Arterial RI measurements by duplex Doppler USG have been advocated for the diagnosis of obstruction. ${ }^{[4]}$ Doppler USG enables detection of subtle intrarenal blood flow changes associated with various pathophysiologic conditions. ${ }^{[5]}$ Platt et al ${ }^{[4]}$ suggested that
Doppler USG is promising for distinguishing obstructive from nonobstructive dilatation in a small group of patients.

Our aim was to evaluate and compare the Doppler waveform alterations in unilateral acute renal obstruction (UARO) with the contralateral normal kidney as a control. We studied the utility of Doppler waveform alterations in UARO patients presenting without dilatation of the pelvicalyceal system (PCS). We also studied the effect of site and degree of obstruction and timing of the Doppler evaluation after onset of symptoms on Doppler waveform alterations.

\section{Materials and Methods}

This prospective case control study was carried at a tertiary care center between May 2005 and March 2006. All patients presenting to the emergency medical division within $24 \mathrm{~h}$ of onset of symptoms of unilateral acute renal colic were part of this analysis. The kidney on the side of obstruction 
was treated as the case kidney and the contralateral normal (unobstructed) kidney served as the control. All patients underwent USG and Doppler USG (HDI 5000, Philips, India) using a 3.5-5 MHz transducer.

Presence or absence of PCS dilatation was assessed in each kidney on the gray-scale images. At least three Doppler spectra were obtained from interlobar arteries along the border of the medullary pyramids and their mean was taken. The Doppler waveforms were made using the lowest pulse repetition frequency possible without aliasing. This maximized the size of the Doppler spectrum and decreased the percentage error in the measurements. In addition, the lowest possible wall filter for each ultrasound scanner was used. The Doppler sample width was set at 2-5 mm. The renal RI was calculated as follows: (peak systolic velocityend diastolic velocity)/peak systolic velocity, with the RI difference (delta RI) determined as the difference in RI of the corresponding and contralateral kidney. Mean RI value was calculated for each kidney.

Intravenous urography or CT scan were the gold standards for demonstrating obstruction. IVU or CT scan were also used to detect the site and degree of obstruction. The site of obstruction was considered to be proximal, if it was up to or proximal to the L3 vertebral level and distal, if beyond. The degree of obstruction was considered complete in cases with delayed excretion of contrast material and partial in patients with prompt excretion of contrast. In patients with a nondilated PCS, increasingly dense nephrogram on IVU or demonstration of calculus on $\mathrm{CT}$, were the criteria used to diagnose obstruction. Those patients not confirmed to have obstruction by IVU or CT were excluded from the analysis. Patients with surgical or medical renal disease were also excluded from this study.

All data was systematically collected, tabulated and analyzed using Microsoft Excel and Strata 6 for Windows. Student $t$-test was used in univariate analysis for continuous variables and Chi square test was used for analysis of noncontinuous data. P value of less than 0.05 was considered to be statistically significant.

\section{Results}

In all, 40 patients (80 kidneys; 40 obstructed kidneys as cases and 40 unobstructed contralateral kidneys as controls) were part of this analysis. Doppler USG was performed in all cases, IVU in 38(95\%) cases and CT scan in $7(17.5 \%)$ cases.

\section{Demographic Profile}

Of 40 patients, 25 were men and 15 women. The mean age (in years) in men, $39.24 \pm 14.12(18-65)$ was higher compared to $34 \pm 14.27(14-55)$ in women. This difference was statistically not significant.

\section{Resistivity Indices}

The mean Resistivity Index (RI) in obstructed kidneys [Figure 1] was significantly higher than in unobstructed kidneys (0.72 Vs 0.64; p <0.001). RI was higher in obstructed kidneys in all the cases. The difference in RI between obstructed and unobstructed kidney (delta RI) ranged from 0.05 to 0.18 with a mean delta RI of 0.08 .

\section{Factors Affecting Resistivity Indices}

\section{Duration of symptoms}

Most of the patients (55\%) were evaluated between 6-12h, $25 \%$ within $13-18 \mathrm{~h}$ and $20 \%$ within $19-24 \mathrm{~h}$ after the onset of symptoms. RI values were similar in all three groups [Table 1]. Thus RI was not a time-dependent parameter in this study.

2. Site of obstruction

The site of obstruction was proximal in 30 (75\%) cases and distal in 10 (25\%) cases. The mean RI of the obstructed kidneys with proximal obstruction was higher $(0.73 \pm$ $0.03)$, than in the kidneys with distal obstruction $(0.72$ $\pm 0.02)$. However, the difference was statistically not significant [Figure 2].

3. Degree of obstruction

Twenty-five $(62.5 \%)$ patients had complete, while 15 $(37.5 \%)$ patients had partial obstruction. RI value in completely obstructed kidneys was significantly higher [Table 2] than partially obstructed kidneys (0.74 Vs 0.70 ; $\mathrm{p}<0.05)$. However, even in partially obstructed kidneys, RI was significantly higher than in the contralateral (unobstructed) kidney. Thus, the degree of obstruction (complete vs partial) was an important parameter affecting the RI value in this study.

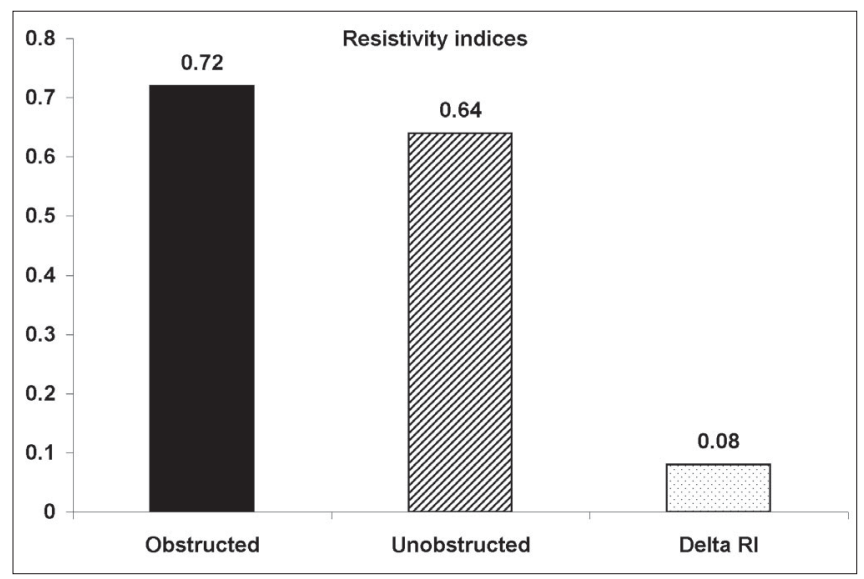

Figure 1: Resistivity indices in obstructed and unobstructed kidney

Table 1: Relationship of resistivity indices with duration of obstruction

\begin{tabular}{lcccl}
\hline & $\begin{array}{c}\mathbf{6 - 1 2} \text { Hrs } \\
\mathbf{N}=\mathbf{2 2}\end{array}$ & $\begin{array}{c}\mathbf{1 3 - 1 8} \text { Hrs } \\
\mathbf{N}=\mathbf{1 0}\end{array}$ & $\begin{array}{c}\mathbf{1 9 - 2 4} \text { Hrs } \\
\mathbf{N}=\mathbf{0 8}\end{array}$ & \\
\hline Obstructed kidney (RI) & $0.73 \pm 0.03$ & $0.71 \pm 0.02$ & $0.73 \pm 0.03$ & NS \\
Unobstructed kidney (RI) & $0.64 \pm 0.03$ & $0.63 \pm 0.01$ & $0.63 \pm 0.04$ & NS \\
Delta RI & $0.09 \pm 0.02$ & $0.08 \pm 0.01$ & $0.10 \pm 0.02$ & NS \\
\hline
\end{tabular}




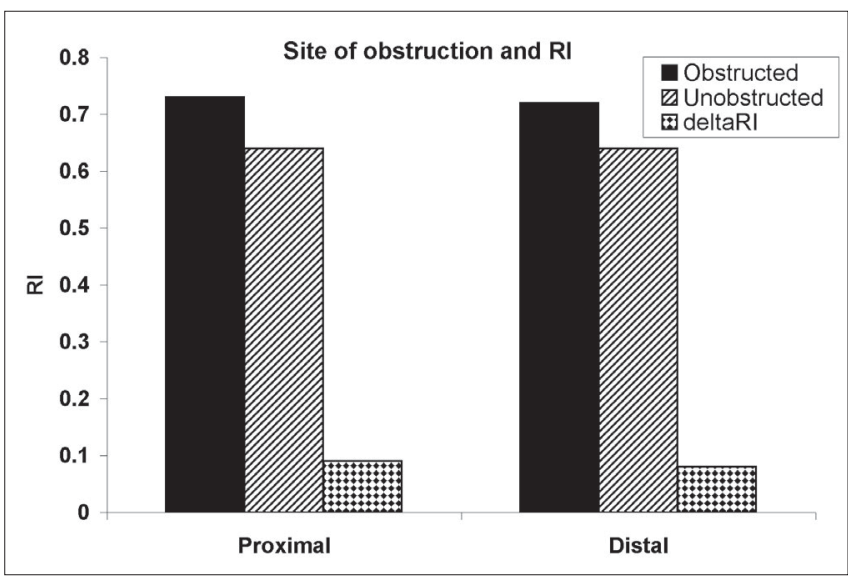

Figure 2: Relationship of resistivity indices with site of obstruction

Table 2: Relationship of resistivity indices with degree of obstruction

\begin{tabular}{lccc}
\hline & $\begin{array}{c}\text { Complete } \\
\mathbf{N}=\mathbf{2 5}\end{array}$ & $\begin{array}{c}\text { Partial } \\
\mathbf{N}=\mathbf{1 5}\end{array}$ & P value \\
& $0.74 \pm 0.03$ & $0.70 \pm 0.02$ & $<0.05$ \\
\hline Obstructed kidney (RI) & $0.63 \pm 0.03$ & $0.64 \pm 0.01$ & NS \\
Unobstructed kidney (RI) & $0.11 \pm 0.02$ & $0.06 \pm 0.01$ & NS \\
Delta RI & \\
\hline
\end{tabular}

\section{PCS dilatation on USG}

On USG, PCS dilatation was observed in 31(77.5\%) patients while it was absent in nine (22.5\%). RI values were similar in both groups. Doppler USG was useful in diagnosing acute renal obstruction even in those cases, where PCS dilatation was absent on USG.

\section{Statistical value of discriminatory threshold of $\mathrm{RI} \geq \mathbf{0 . 7 0}$}

We studied the statistical value of a threshold RI of $\geq 0.70$ in our study. Using this discriminatory value, the overall sensitivity was $87.5 \%$ and specificity $90 \%$. In patients having complete obstruction, the sensitivity increased to $96 \%$, while the specificity was $84 \%$. In patients having partial obstruction, the sensitivity decreased to $73.3 \%$, but the specificity was $100 \%$.

\section{Effects of changes in discriminatory threshold of delta RI}

We also studied the effect on the predictive value of the investigation, of changing the discriminatory threshold of the delta RI. Sensitivity was $95 \%$ and specificity $100 \%$ at a delta RI of $\geq 0.06$. With a higher delta RI, specificity remained unchanged, but the sensitivity fell rapidly [Figure 3]. Thus a delta RI of $\geq 0.06$ was found to be highly sensitive and specific in this study.

\section{Discussion}

Urinary system dilatation seen on conventional gray scale USG has been shown to be sensitive (90\%) but not specific $(65 \%-84 \%)$ in the diagnosis of renal obstruction. ${ }^{[1]}$
Effects of changes in discriminatory threshold of deltaRI

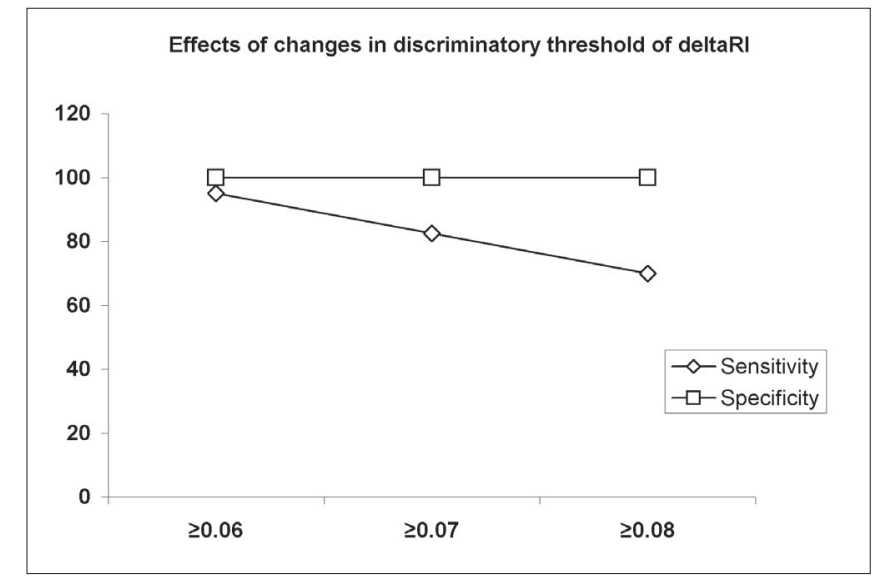

Figure 3: Effects of changes in discriminatory threshold of delta RI

Traditionally, the evidence of renal obstruction provided by USG has been indirect and dependent on the 'anatomical' criterion of dilatation of the PCS and ureter proximal to the level of obstruction. However, USG fails to reveal hydronephrosis in acute obstruction of the kidney in up to $35 \%$ of cases. ${ }^{[1,6,7]}$ More direct 'functional' evidence of obstruction has usually required scintigraphy, ${ }^{[8]}$ but recently, Doppler US techniques have been used to obtain functional information in suspected renal obstruction. ${ }^{[4]}$

USG imaging may miss the diagnosis of obstruction in a variety of situations ${ }^{[9]}$ Mild dilatation may be overlooked or considered clinically insignificant. ${ }^{[10]}$ Some patients with obstructive renal failure may show no PCS dilatation. ${ }^{[11]}$ The reasons for this are unclear; in some patients it may relate to dehydration or to decompression of the pelvicalyceal system by rupture of a calyceal fornix. ${ }^{[8]}$

PCS dilatation may be missed if the PCS system is filled with blood clot, calculus, tumor or pus. ${ }^{[8]}$ Intermittent ureteric obstruction, particularly caused by ureteric calculi, may also lead to a failure to visualize the collecting system with USG. ${ }^{[8]}$

On the contrary, in an attempt not to miss the diagnosis of obstruction in patients with only mild PCS dilatation, the false positive rate of diagnosis may be as high as $26 \% \cdot{ }^{[12,13]}$ Causes of a false-positive diagnosis include: (i) Visualization of a normal PCS system, when there are anatomical variants such as extrarenal pelvis, when the bladder is distended ${ }^{[1]}$ or under conditions of diuresis (ii) Visualization of a dilated but unobstructed system when there is vesico-ureteric reflux (VUR), a distensible system after previous obstruction or infection, dilated calyces (e.g. in papillary necrosis or reflux nephropathy) or during normal pregnancy ${ }^{[14]}$ (iii) Central renal fluid collections other than the PCS, including normal vessels, ${ }^{[15]}$ renal artery aneurysm and peripelvic cysts. ${ }^{[16]}$

Acute unilateral ureteric obstruction results in a complex 
sequence of changes in renal blood flow and ureteric pressure. ${ }^{[17]}$ In the first two hours, the renal blood flow increases, because of afferent arteriole vasodilatation and the ureteric pressure increases. From two to six hours after obstruction, the renal blood flow decreases, secondary to vasoconstriction of the efferent arterioles and the ureteric pressure remains elevated. Subsequently, at six to eighteen hours, the renal blood flow remains reduced, because of vasoconstriction of the afferent arterioles and the ureteric pressure decreases. With bilateral ureteric obstruction, the initial pattern of blood flow change is slightly different, but the resultant decrease in renal blood flow by $24 \mathrm{~h}$ is similar to that in unilateral ureteric obstruction.

The role of renal Doppler USG in the evaluation of acute renal obstruction has been vigorously debated. ${ }^{[18]}$ Rodgers et $a l^{[19]}$ found an elevated RI in acutely obstructed kidneys, especially when compared with the RI in normal contralateral kidneys and with a control group of healthy subjects. Similar results were obtained by Platt et al ${ }^{[2]}$ in 23 patients with acute unilateral ureteric obstruction. However, others have reported that Doppler USG is highly insensitive for detecting acute renal colic. Tublin et a ${ }^{[20]}$ correlated the results of Doppler USG with those of urography in 32 patients presenting with symptoms of renal colic. When the published discriminatory thresholds for obstruction (mean $\mathrm{RI} \geq 0.70$ and $\Delta \mathrm{RI} \geq 0.10$ ) were applied, the sensitivity and specificity of Doppler USG were only $44 \%$ and $82 \%$, respectively.

The application of proper technique is essential for obtaining accurate results in Doppler USG. The most common reason for obtaining a normal RI in the presence of significant obstruction is a technical error. ${ }^{[21]}$ The use of the correct scale (pulse-repetition frequency) to expand the waveform size to fill as much of the available display as possible, without aliasing, is crucial. ${ }^{[22]}$

In our study of 40 patients, we found that the RI in obstructed kidneys was significantly higher than in the unobstructed kidneys (0.72 Vs 0.64; $\mathrm{p}<0.001)$. The RI was higher in obstructed kidneys in all cases. The difference between the obstructed and unobstructed kidneys (delta RI) ranged from 0.05 to 0.18 with a mean delta RI of 0.08 . Our results correlate well with many studies reported earlier. ${ }^{[2,19,23]}$

Previous investigators have shown that the increase in RI occurred after as little as six hours of clinical obstruction. ${ }^{[2]}$ However, admission to hospital in the first five hours after the onset of renal colic is uncommon. ${ }^{[19]}$ We were unable to investigate the shortest duration of acute renal obstruction that can cause elevation of RI, as all the present patients presented with a renal colic of more than six hours duration.
We divided our patients into three groups (6-12h, 13-18h and 19-24h) based on the duration of the renal colic. RI values were similar in all three groups of patients. In patients with renal colic for more than six hours, conflicting results have been obtained with respect to the effect of duration on RI. We agree with Platt et al[ ${ }^{[2]}$ and Shokeir et al ${ }^{[21]}$ that kidneys obstructed for more than $12 \mathrm{~h}$ do not have a significantly higher RI than those with obstruction of shorter duration. On the other hand, de Toledo et al ${ }^{[24]}$ reported that the RI was significantly higher in patients with renal colic of more than $24 \mathrm{~h}$. The variation in the observations could be related to the fact that the duration of obstruction in all these studies was based on clinical symptoms which may not correlate with anatomical obstruction.

We also studied the effect of the level of obstruction on RI values. In our study the level of ureteric obstruction (proximal v/s distal) had no significant impact on the values of RI, in agreement with Platt et al[ ${ }^{[2]}$ and Shokeir AA et al. ${ }^{[21]}$ However, de Toledo et a ${ }^{[24]}$ have shown that patients with proximal ureteric obstruction have RIs higher than those with distal obstruction.

We also looked at the effect of degree of obstruction on RI values. In a recent study, de Toledo et al ${ }^{[24]}$ investigated the diagnostic accuracy of Doppler USG in complete as well as partial acute renal obstruction in 64 patients. With a threshold RI of $\geq 0.70$ and of $\Delta R I \geq 0.06$, they showed a sensitivity of $92 \%$ in 37 patients with complete and $48 \%$ in 27 patients with partial obstruction. Shokeir AA et a ${ }^{[21]}$ showed a sensitivity of $86 \%$ in 42 patients with complete and $62 \%$ in 26 patients with partial obstruction. Our results are similar. The RI value in completely obstructed kidneys was significantly higher than in partially obstructed kidneys (0.74 Vs 0.70; $\mathrm{p}<0.05)$.

We also analyzed the utility of Doppler USG in patients not having dilatation of the PCS on USG. Nine patients, who were later confirmed to have obstruction on IVU, did not have PCS dilatation on USG. RI values were higher in all these patients, with a delta RI of 0.08. Thus, Doppler USG was useful in diagnosing acute renal obstruction even in those cases, where PCS dilatation was absent on USG. The sensitivity of USG in detecting PCS dilatation in acute obstruction was $77.5 \%$ in this study.

Discriminatory RI $\geq 0.70$ and delta RI of $\geq 0.06$ were found to be highly sensitive and specific in this study. Platt JF et $a l^{[2]}$ also found a delta RI of $\geq 0.06$ to be a useful and accurate diagnostic tool.

\section{Conclusions}

Doppler USG is a useful diagnostic tool in unilateral acute renal obstruction. The sensitivity of gray scale USG for 
detecting obstruction was found to be $77.5 \%$. Doppler USG was useful in diagnosing obstruction even when USG findings were normal. The duration of symptoms at presentation or the site of obstruction did not affect the RI values in acute renal obstruction. Patients with complete obstruction had significantly greater RI values than those having partial obstruction. However, even in partial obstruction, RI values were significantly greater than in the contralateral normal kidneys. Using the discriminatory threshold value of $R I \geq 0.70$, the overall sensitivity of Doppler USG in diagnosing acute renal obstruction was $87.5 \%$ and specificity $90 \%$. Using a threshold value of delta RI of $\geq 0.06$, the sensitivity was $95 \%$ and specificity $100 \%$. With higher delta RI, specificity remained unchanged, but the sensitivity fell rapidly. A delta RI of $\geq 0.06$ is a highly sensitive and specific investigation.

\section{References}

1. Ellenbogen PH, Scheible FW, Talner LB, Leopold GR. Sensitivity of gray-scale ultrasound in detecting urinary tract obstruction. AJR Am J Roentgenol 1978;130:731-3.

2. Platt JF, Rubin JM, Ellis JH. Acute renal obstruction: Evaluation with intrarenal duplex Doppler and conventional US. Radiology 1993;186:685-8.

3. Dodd GD 3rd, Kaufman PN, Bracken RB. Renal arterial duplex Doppler ultrasound in dogs with urinary obstruction. J Urol 1991;145:644-6.

4. Platt JF, Rubin JM, Ellis JH, DiPietro M. Duplex Doppler US of the kidney: Differentiation of obstructive from non-obstructive dilatation. Radiology 1989;171:515-7.

5. Platt JF, Rubin JM, Ellis JH. Lupus nephritis: predictive value of conventional and Doppler US and comparison with serologic and biopsy parameters. Radiology 1997;203:82-6.

6. Laing FC, Jeffrey RB Jr, Wing VW. USG versus excretory urography in evaluating acute flank pain. Radiology 1985;154:613-6.

7. Mostbeck GH, Zontsich T, Turetschek K. Ultrasound of the kidney: Obstruction and medical diseases. Eur Radiol 2001;11:1878-89.

8. Webb JA. Ultrasonography and Doppler studies in the diagnosis of renal obstruction. BJU Int 2000;86:25-32.

9. Amis ES Jr, Cronan JJ, Pfister RC, Yoder IC. Ultrasonic inaccuracies in diagnosing renal obstruction. Urology 1982;19:101-5.

10. Rascoff JH, Golden RA, Spinowitz BS, Charytan C. Non-dilated obstructive nephropathy. Arch Intern Med 1983;143:696-8.
11. Millet PJ, Pelle-Francoz DP, Laville M, Gay F, Pinet A. Non-dilated obstructive acute renal failure: Diagnostic procedures and therapeutic management. Radiology 1986;160:659-62.

12. Talner LB, Scheible W, Ellenbogen PH, Beck CH, Gosink BB. How accurate is ultraUSG in detecting hydro-nephrosis in azotaemic patients? Urol Radiol 1981;3:1-6.

13. Webb JA, Reznek RH, White FE, Cattell WR, Fry IK, Baker LR. Can ultrasound and computed tomography replace high-dose urography in patients with impaired renal function? Quart J Med 1984;53:411-25.

14. Fried AM. Hydronephrosis of pregnancy: Ultrasonographic study and classification of asymptomatic women. Am J Obstet Gynaecol 1979;135:1066-70.

15. Scola FH, Cronan JJ, Schepps B. Grade I hydronephrosis pulsed Doppler US evaluation. Radiology 1989;171:519-20.

16. Patel U, Huntley L, Kellett MJ. Sonographic features of renal obstruction mimicked by peripelvic cysts. Clin Radiol 1994;49:4814 .

17. Gulmi FA, Felsen D, et al. Pathophysiology of urinary tract obstruction. In Walsh PC, Reti KAB, Vaughan ED, Wein AJ, eds. Campbell's Urology. 7th ed. Chapt 9. WB Saunders: Philadelphia; 1998. p. 350.

18. Gottlieb RH, Luhman K, Oates RP. Duplex ultrasound evaluation of normal kidneys and native kidneys with urinary tract obstruction. J Ultrasound Med 1989;8:609-11.

19. Rodgers PM, Bates JA, Irving HC. Intrarenal Doppler ultrasound studies in normal and acutely obstructed kidneys. Br J Radiol 1992;65:207-12.

20. Tublin ME, Dodd GD 3rd, Verdile VP. Acute renal colic: Diagnosis with duplex Doppler US. Radiology 1994;193:697-701.

21. Shokeir AA, Abdulmaaboud. Resistive index in renal colic: A prospective study. BJU Int 1999;83:378-82.

22. Platt JF, Ellis JH, Rubin JM. Role of renal Doppler imaging in the evaluation of acute renal obstruction. AJR Am J Roentgentol 1995; 164:379-80.

23. Miletic D, Fuckar Z, Sustic A, Mozetic V, Smokvina A, Stancic $M$. Resistance and pulsatility indices in acute renal obstruction. J Clin Ultrasound 1998;26:79-84.

24. de Toledo LS, Martinez-Berganza Asensio T, Cozcolluela Cabrejas R, de Gregorio Ariza MA, Pardina Cortina P, Ripa Saldias L. Doppler duplex ultrasound in renal colic. Eur J Radiol 1996;23:143-8.

Source of Support: Nil, Conflict of Interest: None declared. 